\title{
FОTO

\section{El uso del zombi como construcción metafórica en la ficción televisiva. El caso de In the Flesh}

\section{The use of zombies as metaphorical constructions in television fiction. In the Flesh as a case study analysis}

\author{
Alberto Añón Lara \\ Universidad de Córdoba, España \\ l92anlaa@uco.es
}

\section{Resumen:}

Desde que en 1968 se estrenara La noche de los muertos vivientes (Night of the Living Dead, George A. Romero, 1968) el zombi ha funcionado como una de las figuras alegóricas más importantes del cine de terror. Ahora que las ficciones televisivas están de moda, convirtiéndose en uno de los fenómenos culturales más notables de los últimos tiempos, el género ha conseguido evolucionar gracias al formato serializado. El potencial metafórico que caracteriza a esta criatura se ha visto revitalizado por la aparición de narrativas que nos invitan a ponernos en su piel, dotándolo de capacidades cognitivas que lo convierten en protagonista de sus propias historias. Nuestro objetivo, por tanto, consiste en estudiar el origen de la humanización del muerto viviente y, por último, analizar la serie In the Flesh (BBC Three: 2013-2014), uno de los casos que mejor han sabido utilizar esta novedosa particularidad.

\begin{abstract}
:
Since the 1968 release of Night of the Living Dead (George A. Romero, 1968), the zombie has worked as one of the most important allegorical figures in horror movies. Now that television fictions are in fashion, becoming one of the most remarkable cultural phenomena of recent times, the genre has managed to evolve thanks to the serialized format. The metaphorical potential that characterizes this creature has been revitalized by the appearance of narratives that invite us to put ourselves in its skin, giving it cognitive abilities that make it the protagonist of its own stories. Our aim, therefore, is to study the origin of the humanization of the living dead and, finally, to analyze the television show In the Flesh (BBC Three: 2013-2014), one of the cases that have made the best use of this innovative feature.
\end{abstract}

Palabras clave: televisión; metáfora; zombi; homofobia; George A. Romero; In the Flesh

Keywords: Television; Metaphor; Zombie; Homophobia; George A. Romero; In the Flesh 


\section{Introducción}

Durante la década de 1980 el género zombi entró en una etapa de decadencia en lo relativo a su potencial metafórico, aquel que tantos éxitos había granjeado a George A. Romero. Hasta este momento, al no estar sujeto a la complejidad propia de unos antecedentes literarios, el zombi se había erigido en lo que Manning llama un texto en blanco, “[...] a space waiting to be filled by both the universal and the historically specific contemporary anxieties of us ordinary people, occupying our mundane spaces in the home, workplace, community or shopping mall.” (Manning, 2015, p.164). El zombi, en definitiva, siempre ha servido como justificación para criticar aspectos que nos conciernen como sociedad o para reflejar nuestros miedos. Este debilitamiento del zombi como construcción metafórica mediante la cual sacar a relucir problemas sociales y políticos de diversa índole, curiosamente, comenzó a mediados de los ochenta tras el estreno de El día de los muertos (Day of the Dead, George A. Romero, 1985), película con la que el director norteamericano daría cierre al tríptico iniciado por La noche de los muertos vivientes y continuado con Zombi (Dawn of the Dead, George A. Romero, 1978).

Las películas de la época abandonarían así el modelo de zombi concebido por Romero y comenzarían a satisfacer las demandas de un público que, en plena edad dorada del slasher, buscaba en el cine de terror una mayor explotación del sexo y de la violencia. Por este motivo, muchos de los estudios que han abordado la figura del muerto viviente en el cine han denostado este periodo de su historia cinematográfica, olvidando de manera injustificada que en ella se experimenta e introducen algunas de las novedades más influyentes en el desarrollo del género en la actualidad, sobre todo en lo relativo a las ficciones televisivas.

Partiendo de esta premisa, proponemos adentrarnos en las principales características que durante esta etapa se introducen en el cine zombi, entre las cuales destaca la dotación de capacidades cognitivas al muerto viviente, haciendo de él un monstruo más humano. Esto tendrá como resultado la aparición de narrativas que explorarán los beneficios potenciales y las 
problemáticas derivadas de ser un zombi. Como consecuencia del éxito mundial de series como The Walking Dead (AMC: 2010-) o su spin-off Fear the Walking Dead (AMC: 2015-), es en las ficciones televisivas donde encontraremos un mayor uso del zombi como una criatura capaz de sentir y con la que poder empatizar. Las series de televisión son las que juegan en mayor grado con la transgresión del hasta entonces canon que regía el género, acto seguido veremos en qué formas esta humanización de la que hablamos se nos presenta dentro de uno de los fenómenos culturales más importantes del siglo XXI. De entre todos los ejemplos, nuestro foco se centrará en el análisis de la serie británica In the Flesh (BBC Three: 2013-2014), por tratarse de una de las propuestas más interesantes de los últimos años al utilizar la humanización del zombi para construir una crítica en torno a la homofobia en el contexto de una comunidad dominada por el fundamentalismo religioso y político.

\section{Las innovaciones de las comedias zombis: profundizando en la humanización del muerto viviente}

Negar la influencia de Zombi es difícil, el regreso de Romero al monstruo que tantas alegrías le había dado fue determinante en el devenir del género. La tan esperada secuela de La noche de los muertos vivientes consiguió encumbrar al zombi hasta puntos insospechados, llegando a crear una corriente en países europeos, sobre todo España e Italia, que aprovecharon la estela del filme de Romero para producir una considerable cantidad de películas sobre zombis. La diferencia es que estas películas prestan una menor importancia a la utilización del zombi como artefacto cultural y hacen un uso aún más excesivo de la violencia y del gore. Lo llamativo es que el público norteamericano, a partir de mediados de la década de los ochenta, comenzaría a demandar un nivel de violencia similar, así como un tono más cómico (Bishop, 2010, p.186). Esto se va a traducir en el inicio de una etapa en la que el zombi aparecerá, salvo contadas excepciones, en clave de comedia, desprovisto de la más mínima intencionalidad crítica. 
Este viraje en las preferencias de los aficionados quedó plasmado en la escasa acogida de la tercera contribución de Romero al género. Si Zombi había alcanzado un éxito enorme, cimentando las bases del cine zombi, El día de los muertos fracasó estrepitosamente en taquilla y el zombi comenzó a perder, como decimos, casi la totalidad de su componente metafórico. Más esclarecedor todavía es el hecho de que El regreso de los muertos vivientes (The Return of the Living Dead, Dan O'Bannon, 1985), una de las grandes comedias zombis de la época, estrenada el mismo año que la de Romero, superara con creces la recaudación de esta última ${ }^{1}$. Sin embargo, independientemente de la pérdida de su capacidad para reflejar las ansiedades sociales, no podemos pasar por alto que ya en la década de los ochenta el zombi "[...] had established too strong a cult following to suffer much from this hiatus [...]" (Dendle, 2007, p.53). Su tradición era bien conocida por la sociedad norteamericana y, de hecho, podemos considerar que estaba ya integrado como uno de los iconos más destacables de la cultura popular del momento. Precisamente, fue su fuerte raigambre en la cultura popular lo que garantizó su supervivencia en una de las etapas más duras de su historia cinematográfica; especialmente en la década de 1990, cuando el número de producciones disminuye drásticamente y no se recuperará hasta principios del siglo XXI. Hagamos un inciso para ver algunos ejemplos sobre esta cuestión.

Uno de los casos más representativos lo encontramos en el mundo de la industria musical, con el videoclip de la canción Thriller (1983) de Michael Jackson. Este videoclip, dirigido por el director de cine John Landis, se erige como una parodia autorreferencial de los estereotipos, códigos y convenciones del cine de terror. Dejando a un lado la escasa relación entre la letra de la canción y el thriller como género cinematográfico, el videoclip muestra una clara intencionalidad narrativa, con un inicio y un final bien definidos (Mercer, 1993, pp.96-97). A pesar de ello, la trama es inexistente, ya que todo está supeditado, como decimos, a esa parodia de la tradición cinematográfica del

\footnotetext{
1 Más información sobre la recaudación de El día de los muertos en: https://www.boxofficemojo.com/movies/?id=dayofthedead.htm (Consulta: 28-09-2018). Más información sobre la recaudación de El regreso de los muertos vivientes en: https://www.boxofficemojo.com/movies/?id=returnofthelivingdead.htm (Consulta: 28-092018).
} 
cine de terror. En él aparecen multitud de alusiones al género, pero podemos destacar dos momentos clave del videoclip, aquellos en los que Michael Jackson sufre una transformación: la primera en hombre lobo y la segunda en zombi; durante esta última tiene lugar una de las coreografías más emblemáticas de la música pop.

Los videojuegos también jugaron un papel fundamental en la supervivencia y propagación del zombi en la cultura popular en la década de los ochenta y, sobre todo, en los noventa. Concretamente en 1996, la compañía nipona Capcom lanzó para la plataforma PlayStation de Sony la primera entrega de Resident Evil, que acabaría dando lugar a múltiples secuelas y convirtiéndose así en una de las sagas más prolíficas de la historia de los videojuegos. Así mismo, tal fue la popularidad cosechada por la mítica saga de Capcom que esta fue adaptada al cine en el año 2002, dejando un total de seis entregas (20022016) y haciendo de las películas del universo Resident Evil la franquicia basada en videojuegos de mayor éxito de todos los tiempos (Tartaglione, 2017).

La presencia del zombi también fue intensa en televisión, apareciendo principalmente en series de animación a modo de parodia, como en South Park (Comedy Central: 1997-)² o la franquicia Scooby-Doo3, que continúa emitiéndose de forma casi ininterrumpida desde 1969 en diferentes cadenas y canales de televisión. Pero los mejores ejemplos los encontramos en Los Simpson (The Simpsons, Fox: 1989-), serie que desde sus orígenes ha retratado satíricamente a la sociedad contemporánea, sobre todo a la sociedad estadounidense. En sus episodios semanales, Los Simpson hacen un uso muy rico de referencias, explícitas e implícitas, a iconos de la cultura popular del pasado y del presente (Enríquez Veloso, 2012). Estas referencias, en cualquier caso, se integran dentro de la fórmula tradicional de la serie, cosa que no ocurre en los capítulos especiales que se empezaron a emitir anualmente en

\footnotetext{
2 En el séptimo episodio de la primera temporada (emitido el 29 de octubre de 1997), titulado Pinkeye, un accidente en la morgue convierte a los residentes de South Park en zombis. Hay un momento en el capítulo en el que un personaje afroamericano se convierte en zombi y empiezan a bailar Thriller de Michael Jackson.

3 El 22 de septiembre de 1998 se lanzaba en vídeo la película Scooby-Doo on Zombie Island. En ella la pandilla de Scooby-Doo acaba resolviendo un misterio en la Moonscar Island de Louisiana, donde tienen que hacer frente a un grupo de zombis.
} 
Halloween desde la segunda temporada, la cual comenzó en octubre de 1990 (Jones, 2010, p.56). Los Treehouse of Horror, a diferencia de los episodios normales, son episodios antológicos que se dividen en tres historias diferentes en vez de seguir una única trama, pero manteniendo su tono satírico tan característico. En cada una de estas historias, la conexión con Halloween se hace a través de una gran variedad de alusiones a clásicos de la literatura y del cine de terror, referencias que, como decimos, se apoderan de toda la trama y constituyen la historia en sí (Jones, 2010, pp.62-63).

Así, el zombi aparece de manera recurrente en varios de estos capítulos especiales. La primera de estas apariciones tiene lugar en "Dial ' $Z$ ' For Zombies”, una de las historias del Treehouse of Horror III emitido en 1992. Un año más tarde, en el Treehouse of Horror IV, la familia Simpson es convertida en zombis durante el gag del sofá que da inicio a cada capítulo. También hará acto de presencia esta criatura en los especiales de los años 2002 y 2009, parodiando en este último películas como 28 días después (28 Days Later, Danny Boyle, 2002) y Bienvenidos a Zombieland (Zombieland, Ruben Fleischer, 2009).

Todos estos ejemplos que acabamos de ver, que tienen en común el uso del zombi como elemento paródico, ponen de manifiesto el peso que este monstruo tenía por aquel entonces dentro de la cultura popular. Aclarada esta cuestión, ha llegado el momento de replantearnos cómo afecta el éxito de las comedias zombis al género y, en consecuencia, al desarrollo de la metáfora zombi. En primer lugar, debemos tener en cuenta que desde que Romero estrenara en 1968 La noche de los muertos vivientes, reformulando el género por completo, el zombi ha mantenido una forma más o menos estable. Esta estabilidad se ha mantenido tan fuerte que, inevitablemente, la imagen mental que tenemos de él sigue siendo la del muerto viviente romeriano, es decir, una criatura lenta, antropófaga y sin ninguna capacidad cognitiva aparente. Algo normal si pensamos que la gran mayoría de películas y ficciones televisivas siguen representando al zombi de acuerdo al modelo establecido hace 50 años por Romero. Pero su naturaleza formal, así como su capacidad metafórica, nunca han sido inmutables. De tal forma que, a partir de la década de los ochenta, películas como la ya citada El regreso de los muertos vivientes o Re- 
Animator (Stuart Gordon, 1985) comienzan a transgredir y experimentar con las características del zombi, avanzando algunos de los puntos claves del género en el futuro.

Lo primero que vamos a ver es que ambas películas van a romper con el principio básico establecido por Romero de que para acabar con un zombi es necesario destruir su cabeza. Los muertos vivientes van a ser mutilados de miles de formas y van a seguir moviéndose, llegando incluso a seguir en movimiento cualquiera de sus extremidades corporales4. Una mayor complicación a la hora de destruirlos implica, en consecuencia, un incremento de la violencia gráfica y del gore, algo que apuntábamos al principio de este apartado como una de las características más reclamadas por el público norteamericano.

La segunda aportación que hay que tener en cuenta es que el zombi, como consecuencia de ese incremento de la violencia ejercida hacia él, va a mostrarse como un ser vulnerable, capaz de sufrir y expresar sus emociones, ganándose la simpatía del público; en definitiva, se va a humanizar. Sin embargo, hemos de tener en cuenta que esta humanización fue ya iniciada por Romero en El día de los muertos, momento a partir del cual el muerto viviente va a ir adquiriendo capacidades cognitivas que hasta entonces le eran negadas por la condición de su propia naturaleza. Serán, eso sí, las comedias de los ochenta las que exploten este proceso de humanización y doten al zombi de un mayor protagonismo. Este es un proceso similar al que experimentó el vampiro a raíz de la publicación de novelas como Entrevista con el vampiro (1976) de Anne Rice, y que acabaría teniendo su paralelismo en el cine. A partir de entonces no es raro encontrarnos con narrativas en las que el vampiro mitiga su monstruosidad a través del desarrollo de una conciencia que le impide, entre otras cosas, alimentarse de sangre humana (Tenga \& Zimmerman, 2013, p.76). Hasta tal punto ha llegado su humanización que en muchas de estas ficciones vamos a encontrar ideas tan variopintas como

4 Podríamos citar varios ejemplos al respecto, pero uno de los más representativos lo encontramos en Re-Animator cuando Megan Halsey, personaje interpretado por Barbara Crampton, es abusada sexualmente por la cabeza decapitada del Dr. Carl Hill. 
humanos que fantasean con ser convertidos en vampiros o, incluso, relaciones amorosas humano-vampiro.

Algo similar ocurrirá con el zombi a lo largo del siglo XXI, ya que a medida que las "[...] zombie narrative continues to develop and change, it increasingly challenges the customary definition of 'monster', often exploring the potential benefits of being a zombie." (Bishop, 2015a, p.26). En este sentido, el interés por que sean capaces de pensar y actuar por sí mismos, aunque considerado hasta entonces como una transgresión del canon que había regido el género durante décadas, se demostrará fundamental con el resurgir del género a principios del nuevo siglo. Esto no quiere decir que las nuevas generaciones no vayan a estar interesadas en aquellas narrativas más próximas a las películas clásicas de Romero, pero es cierto que los directores continuarán experimentando con lo que Bishop llama "zombie subjectivity and sentience" y así irán introduciendo novedades (Bishop, 2010, p.186). La humanización del muerto viviente, además, dejará de ser un impedimento, tanto en películas de tono más serio como en comedias, a la hora de elaborar historias en las que poder articular una crítica de corte sociopolítico a las que tan acostumbrados nos tenía el género y que, desgraciadamente, en los años ochenta y noventa caen en desudo. Ahí tenemos ejemplos como Zombies Party (Una noche... de muerte) (Shaun of the Dead, Edgar Wright, 2004), en la que se acaba explorando la idea de la domesticación de los muertos vivientes. Se trata de una comedia romántica que, a grandes rasgos y funcionando desde un conocimiento profundo de la tradición cinematográfica del zombi, abraza la función alegórica empleada por Romero en Zombi, con la diferencia de que aquí, en el intento por elaborar esa crítica al sistema capitalista, se señala que la rutinaria vida de la clase obrera convierte a esta, de facto, en zombis.

Esto, en cualquier caso, no hubiera sido posible sin el inestimable trabajo de Romero primero en El día de los muertos, como hemos mencionado antes, y después en La tierra de los muertos vivientes (Land of the Dead, George A. Romero, 2005), demostrando que en su cine siempre ha habido una clara preocupación por que el espectador empatice con los zombis. En consecuencia, el género se abrirá a nuevas lecturas más complejas, en las que se nos invita a ponernos en la piel de "el Otro". Como veremos, no es que apuesten solamente 
por introducir zombis con alguna capacidad cognitiva, sino que van a presentar narrativas en las que los propios zombis cuentan sus historias, actuando como protagonistas e incluso como héroes.

\section{La adaptación de la metáfora zombi a las ficciones televisivas}

Desde la óptica cultural, las series de televisión se encuentran en su momento más álgido. En su legitimización cultural hay un momento clave que muchos estudios identifican como el punto de inflexión a partir del cual se produce un cambio histórico que repercute de forma significativa en la popularidad cultural de las series. Este punto no sería otro que el estreno de Los Soprano (The Sopranos, HBO: 1999-2007), serie creada por David Chase. La realidad demuestra que la serie creada por David Chase, si bien no fue la primera en hacerlo, sí es la que de manera más patente consiguió aproximar los valores de producción de la ficción televisiva a los del cine. Así mismo, estableció cambios significativos en lo que respecta a los modelos narrativos, demostrando que desde la televisión también se podían alcanzar unas cotas de calidad narrativa similares a las de cualquier filme. El canon de calidad que consiguió implantar se convirtió rápidamente en el modelo imperante, inaugurando una etapa de prosperidad cultural sin precedentes para las series de televisión (Rogers, Epstein, \& Reeves, 2002, p.53).

La serie de HBO tardó poco tiempo en erigirse como estandarte de lo que una serie podía y debía llegar a ser, su transcendencia fue y sigue siendo de una magnitud enorme. En este sentido, las excelentes críticas cosechadas por Los Soprano trajeron consigo a las series de televisión el reconocimiento social y cultural que tan normalizado tenemos hoy en día, reconocimiento que también se hizo extensible al mundo académico, el cual comenzó a incluir en su agenda, en un grado de importancia similar al otorgado tradicionalmente al cine, el estudio de las series de televisión. Casi dos décadas después de su estreno, las series de televisión se han convertido en uno de los fenómenos culturales más importantes del siglo XXI. Las series forman parte de nuestro día a día y gran parte de que esto sea posible se debe a que el acceso a ellas se ve facilitado por las plataformas de video bajo demanda, cuyo número es cada vez mayor. 
En este contexto, el lugar que ocupa el zombi en el actual panorama cultural de las series de televisión es un lugar de preeminencia, dominado en gran medida por The Walking Dead y su spin-off Fear the Walking Dead. La serie de AMC, basada en el cómic homónimo creado por Robert Kirkman en 2003 y que hasta la fecha lleva publicados un total de 184 números, ha batido multitud de records en términos de audiencia (O'Connell, 2014 y 2016), demostrando así la plena vigencia de los muertos vivientes dentro del fenómeno de la serialidad contemporánea. Estos datos de audiencia, lejos de ser un hecho anecdótico, son especialmente significativos en tanto que van a permitir la progresiva aparición de múltiples narrativas dedicadas al zombi, algunas de las cuales aportarán novedades muy interesantes al género y, en consecuencia, al potencial metafórico de este monstruo. Tenemos el caso de Dead Set: Muerte en directo (Dead Set, E4: 2008) de Charlie Brooker, creador de Black Mirror (Channel 4: 2011-2014; Netflix: 2016-), en la que la invasión zombi de la casa de Gran Hermano es utilizada para criticar el creciente consumo de reality shows; la propuesta de Les Revenants (Canal+: 2012-) y su reflexión en torno al dolor y el luto tras el regreso a la vida de un familiar fallecido, y otras como iZombie (The CW, 2015-) o ZombieLars (NRK Super: 2017-). Esta última es de las más recientes y llamativas porque utiliza al zombi, así como a otros seres fantásticos, como elemento pedagógico - la serie está orientada a un público infantil - para concienciar a los más jóvenes sobre el acoso y la discriminación en el ámbito escolar.

En cualquier caso, tal y como apuntábamos en la introducción de este artículo, el siguiente apartado lo dedicaremos al análisis de la miniserie británica In the Flesh, creada por Dominic Mitchell, una de las aportaciones al género más atractivas de los últimos años.

\subsection{En la piel de "el Otro". In the Flesh y su crítica a la homofobia}

Dejando atrás las visiones pesimistas de Romero sobre diversos aspectos de la sociedad norteamericana, In the Flesh apuesta por el uso del punto de vista del zombi "[...] to critique homophobia and the masquerade of gender, sexuality and identity." (Abbot, 2016a, p.174). La serie se sitúa argumentalmente varios años después del apocalipsis zombi. Al contrario de 
lo que ocurre en las narrativas tradicionales, estos muertos vivientes (a los que de forma despectiva llaman "rotters" o podridos) no pueden transmitir su condición a través de la mordedura ni de la sangre, por lo que su amenaza fue contenida de manera más o menos rápida. Así mismo, el gobierno ha encontrado un tratamiento que devuelve a los afectados por el Síndrome del parcialmente muerto (del inglés PDS: Partially Deceased Syndrome) a su estado natural. Gracias a este tratamiento experimental, que debe ser inyectado a diario en la columna vertebral de los PDS, los infectados recuperan sus capacidades cognitivas, así como sus recuerdos de cuando eran humanos. El objetivo del gobierno no es otro que el de reintegrar en la sociedad a estos zombis, si bien esto causará una enorme polémica entre los supervivientes de El Levantamiento (The Rising); así es como se conoce el día en el que los muertos salieron de sus tumbas en busca de carne humana de la que alimentarse.

La narrativa se centra, como decimos, en el uso de la zombificación como símbolo de estigmatización social e intolerancia (Aiossa, 2018, p.152). Estos estigmas los sufrirá, sobre todo, aunque no será el único, Kieren Walker, protagonista principal de In the Flesh; por tanto, la historia es contada fundamentalmente desde su perspectiva. El episodio piloto recoge su proceso de rehabilitación como afectado por el PDS y su regreso a Roarton, su ciudad natal. Roarton es una pequeña comunidad del norte de Inglaterra que lideró la formación de un grupo paramilitar llamado Cuerpo de Voluntarios Humanos (del inglés HVF: Human Volunteer Force), creado por la población local para protegerse de los zombis durante El Levantamiento. Su animadversión por ellos es enorme y, como se puede deducir, no verán con buenos ojos las medidas adoptadas por el gobierno relativas a la reintegración de los PDS a su comunidad.

Con esta premisa, el episodio piloto nos dejará claro desde un primer momento, mediante el uso de planos y puntos de vista en primera persona, que Kieren Walker es la figura central de In the Flesh. El primer episodio comienza con la imagen de una adolescente, vestida con atuendo militar, recogiendo suministros en un supermercado. La normalidad se ve interrumpida cuando la joven se encuentra con una zombi alimentándose de 
uno de sus compañeros de los HVF. Ella le disparará en el pecho y luego intentará huir, con la mala fortuna de que las luces se apagan en ese momento. $\mathrm{Al}$ darse la vuelta, la muchacha se encuentra cara a cara con otro zombi, que le agarra del cuello y, en ese instante, se escucha una voz en off pidiendo a Kieren que se calme. La secuencia se corta y da paso a un plano en el que vemos a un joven, en apariencia convulsionando y gimiendo, que agarra de las solapas de su bata blanca a un hombre que continúa tranquilizándole (F1).

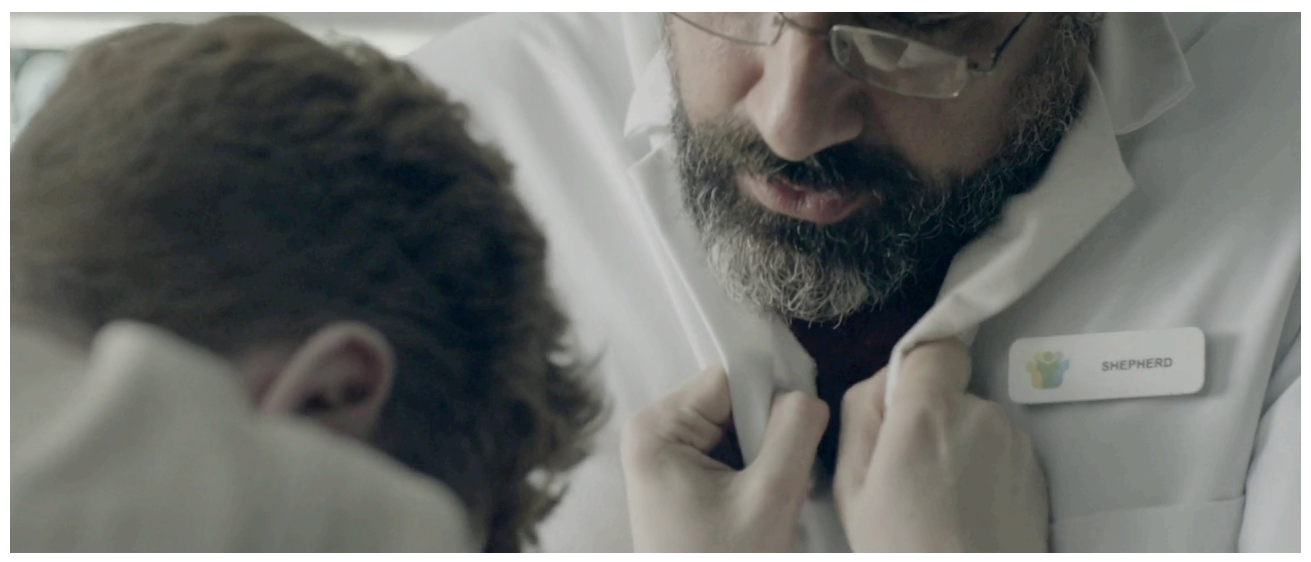

F1. In the Flesh (BBC Three: 2013-2014).

A medida que este se calma, la secuencia se reduce a un primer plano frontal de él mientras levanta la cabeza lentamente hacia la cámara, imagen a la que va a suceder otro primer plano, de nuevo en la oscuridad del supermercado, en el que vemos el rostro de Kieren antes de recibir su tratamiento como PDS. La imagen se funde entonces con la cara de Kieren en la actualidad, en el interior de una consulta del centro médico en el que se encuentra internado (F2).

Sin embargo, el punto de vista de Kieren no es el único que se destaca en este primer episodio. Las escenas de Kieren en el centro médico, un lugar deshumanizado en el que tratan a sus pacientes como ganado (F3), van a alternarse con imágenes de la comunidad de Roarton en las que aparecen pintadas que muestran el odio exacerbado que tiene la población local hacia los zombis, así como otras exaltando a los HVF (F4). 


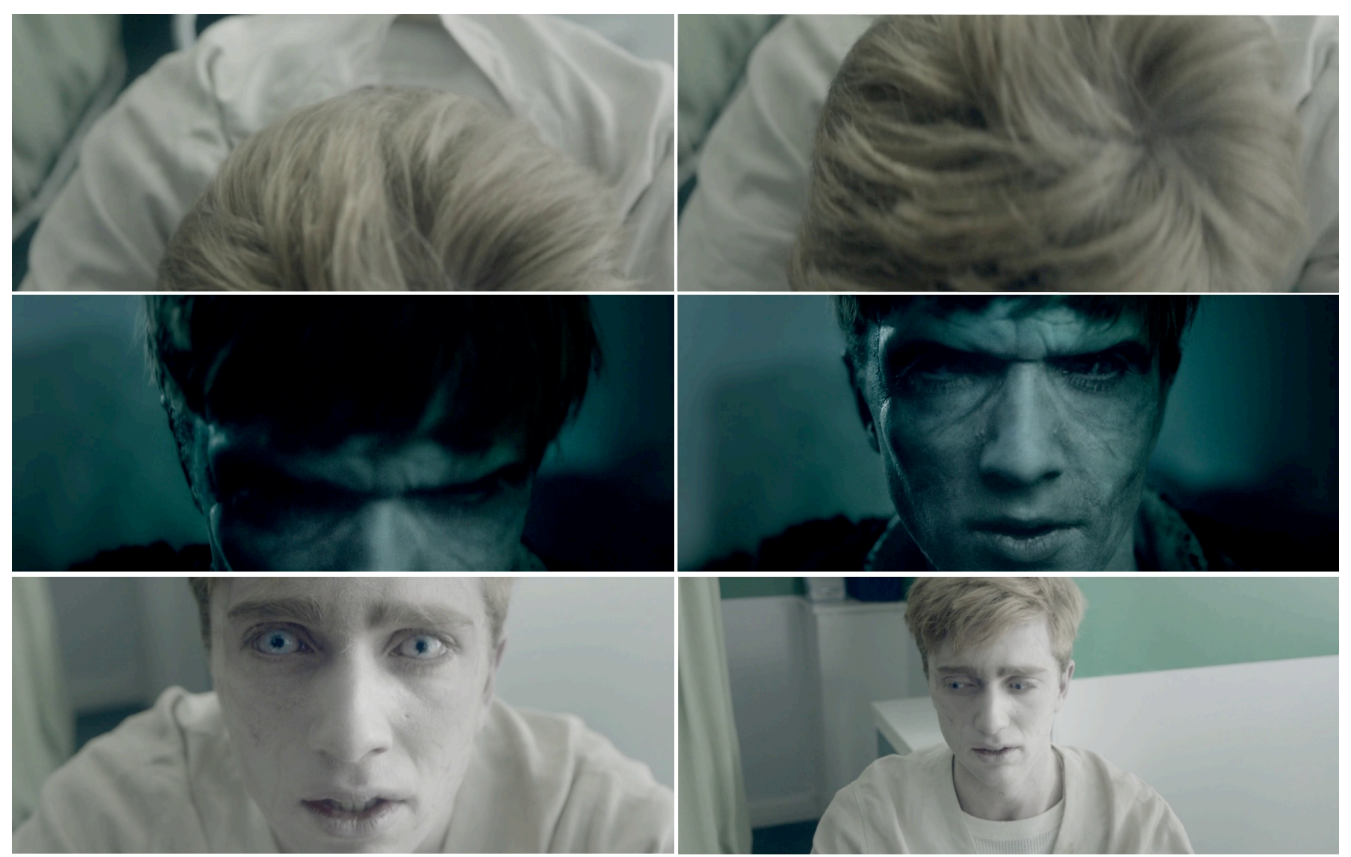

F2. In the Flesh (BBC Three: 2013-2014).

Mientras Kieren se prepara para volver a su hogar, expresando su culpabilidad en terapias de grupo con otros afectados por el PDS, sus padres intentan vender su casa por miedo a posibles represalias que pudieran tomar contra su hijo. Su temor se ve justificado con una escena que tiene lugar poco después, en la que vemos a Bill Macy, líder de los HVF, repartiendo tareas de patrulla a sus subalternos y diciéndoles que tengan especial cuidado porque, aunque el gobierno dice lo contrario, en el bosque sigue habiendo "rotters" o podridos, en referencia a los zombis. En cuanto a los que se medican y están volviendo a las ciudades, Bill Macy dice: "Un podrido es un podrido, con droga o sin ellas. Si hay algunos rondando nos encargaremos de ellos." Curiosamente, entre sus miembros se encuentra Jem, la hermana pequeña de Kieren, quien oculta intencionadamente que su hermano va a volver a la ciudad. El clima que se vive en Roarton es muy hostil. Lo que al principio se nos muestra como un mero indicio quedará probado al finalizar el episodio piloto, cuando Bill Macy saca de su casa a altas horas de la madrugada a una anciana que sufre el PDS y la ejecuta públicamente delante de su marido. 


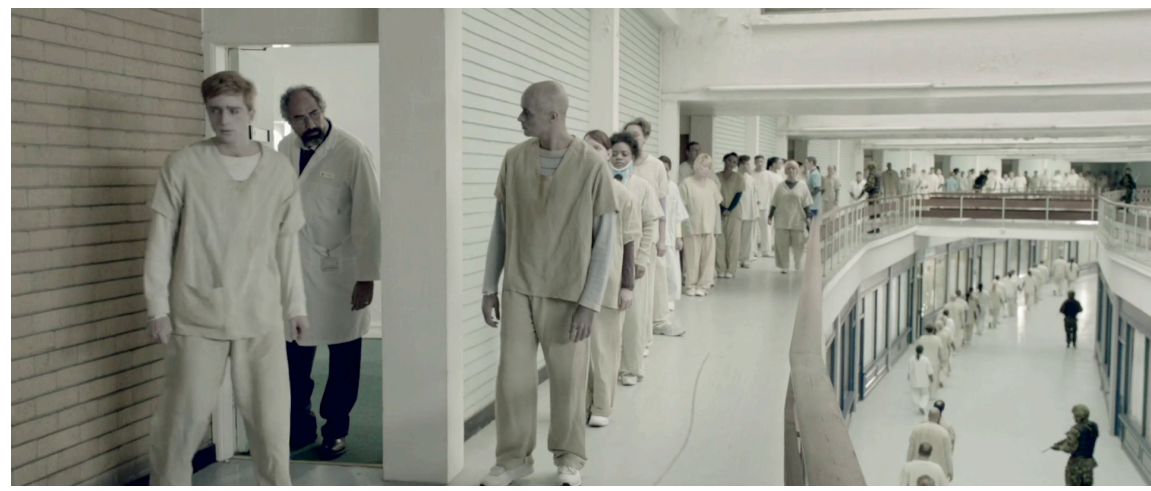

F3. In the Flesh (BBC Three: 2013-2014).

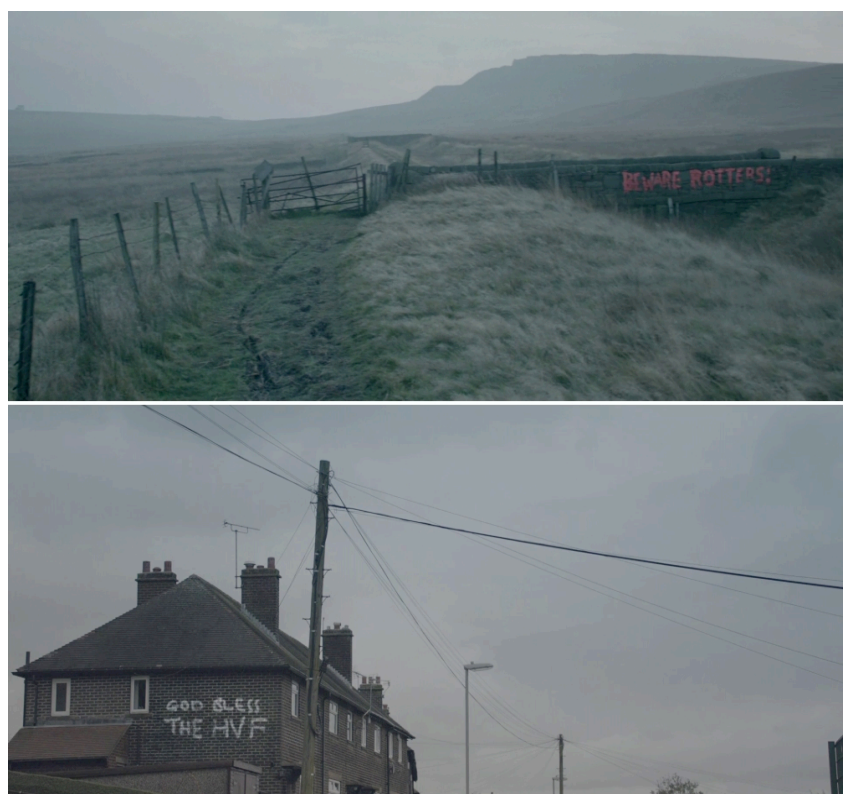

F4. In the Flesh (BBC Three: 2013-2014).

Este contraste entre el centro médico y Roarton del que hablamos se hace también extensible, visualmente, a la forma en la que se nos presenta la culpa y tormento que siente Kieren al recordar el último asesinato que cometió cuando no había recibido aún su tratamiento. Si recordamos, este asesinato aparece recogido en la secuencia del supermercado descrita hace apenas unas líneas. Ya aquí nos damos cuenta de que el contraste entre los flashbacks del pasado de Kieren y las imágenes de su presente es muy revelador. Un ejemplo perfecto lo encontramos en esa misma secuencia, cuando vemos por primera vez su rostro durante su estado zombi e, inmediatamente después, los cambios que experimenta su fisonomía tras recibir el tratamiento que lo devuelve a la normalidad. Esta pauta estética cumple una doble función, como iremos viendo a lo largo de este análisis. Por un lado, sirve para alinear visual y 
emocionalmente al público con Kieren a lo largo de su transformación de zombi a joven incomprendido (Aiossa, 2018, p.155), y, por el otro, remarca ese estigma que supone ser zombi, un estigma que ni el maquillaje ni las lentillas que obligan a usar a los PDS en su regreso a casa pueden ocultar. Al final, In the Flesh lo que hace es utilizar al zombi como metáfora de la homosexualidad y la intolerancia social, por lo que la serie se lee mejor a través de la Teoría Queer (Bishop, 2015b, p.185).

\subsubsection{La homosexualidad y lo monstruoso. ¿Dos términos equivalentes?}

Los estudios dedicados a la representación de la sexualidad en el cine de terror son muy variados y, sin duda, una pieza clave a la hora de abordar narrativas como In the Flesh. En este sentido, uno de los más destacados es el libro Monsters in the Closet: Homosexuality and the Horror Film (1997) de Harry M. Benshoff, un libro que sienta las bases de la relación entre el monstruo en el cine de terror y la homosexualidad, así como sus implicaciones sociopolíticas. En el momento de su publicación, Benshoff consideraba que numerosos sectores de la sociedad norteamericana seguían asociando la homosexualidad a prácticas sexuales deplorables moralmente y al contagio del sida. De acuerdo con sus planteamientos, extensibles a muchos otros países, él considera que en el cine de terror "[...] monster is to 'normality' as homosexual is to heterosexual." (Benshoff, 1997, p.2). Partiendo de esta base, plantea un análisis y revisión del monstruo, entendido como una construcción metafórica que sustituye a la figura del homosexual, en un conjunto de películas de cine de terror a lo largo de múltiples periodos históricos. Algo totalmente normal si abrazamos, de una vez por todas, la idea de que el género del terror, y en especial el monstruo como figura metafórica, es capaz de albergar y codificar mensajes de índole social y política como ningún otro género cinematográfico o televisivo, permitiendo así dar respuesta o, al menos, entender, desde una perspectiva casi pedagógica, determinadas problemáticas sociales. Una hipótesis de partida que Jeffrey Jerome Cohen, un año antes que el propio Benshoff, desarrolla con gran profusión en Monster Theory: Reading Culture (1996) y que el crítico cinematográfico Robin Wood anticipa a mediados de la década de los ochenta en Hollywood from Vietnam to 
Reagan (1986). Al respecto, creemos necesario recuperar las siguientes palabras de Robin Wood, pues entendemos que su postura es muy clarificadora respecto a la cuestión de la que hablamos:

[...] a simple and obvious basic formula for the horror film: normality is threatened by the Monster. [...] Although so simple, the formula provides three variables: normality, the Monster, and, crucially, the relationship between the two. The definition of normality in horror films is in general boringly constant: the heterosexual monogamous couple, the family, and the social institutions (police, church, armed forces) that support and defend them. The Monster is, of course, much more protean, changing from period to period as society's basic fears clothe themselves in fashionable or immediately accessible garmentsrather as dreams use material from recent memory to express conflicts or desires that may go back to early childhood. (Wood, 2003, p.71).

Para Wood, en definitiva, la aparición del monstruo como ruptura del orden establecido (normalidad) es el principio básico por el que se rige el cine de terror. Una idea que entronca, a su vez, con el concepto de "el Otro" y su represión. La Otredad representa, a grandes rasgos, aquello que no debe ser reconocido o aceptado, pero a lo que se debe hacer frente de dos maneras: “[...] either by rejecting and if possible annihilating it, or by rendering it safe and assimilating it, converting it as far as possible into a replica of itself." (Wood, 2003, pp.65-66).

Así, Kieren encarna el papel de "el Otro” y la zombificación (monstruosidad) no es más que un símbolo de su homosexualidad, lo que explicaría por qué todas las palabras utilizadas para describir a los muertos vivientes "[...] contribute to their conceptual (and, at times, literal) separation from the living, whom are understood as the norm from which the undead deviate." (Jackson, 2017, p.130). Homosexualidad que deriva, por un lado, en la opresión a la que es sometido por los habitantes de Roarton antes y después de convertirse en zombi y, por el otro, en la represión interna que le lleva a suicidarse tras conocer que Rick, hijo de Bill Macy y de quien estaba enamorado, ha muerto en Afganistán. Será en su nueva vida como zombi cuando comience a liberarse del yugo de su propia represión y, en consecuencia, a hacer frente a la opresión social. Esta opresión, como vemos a 
lo largo de la serie, funciona a varios niveles. Uno de ellos lo encontramos en el hecho de que se obligue a los zombis a ocultar los signos de su identidad con maquillaje, el cual, como apunta Stacey Abbot, es "[...] is equated within the series with the act of performing heteronormativity or homonormativity -in which homosexuality is made 'acceptable' by mirroring the conventions of heterosexuality." (Abbot, 2016a, p.172). Esto queda patente a medida que avanza la serie y se va haciendo más evidente que Kieren es gay y sus problemas en Roarton comenzaron antes incluso de ser un zombi. Durante su vida como humano, por ejemplo, fue expulsado de por vida del pub local tal y como le explica a su amiga Amy, también afectada por el PDS, en el segundo episodio de la primera temporada: "La gente ahí dentro...me odiaba antes de que fuera de esta manera [un zombi], porque no era como ellos”.

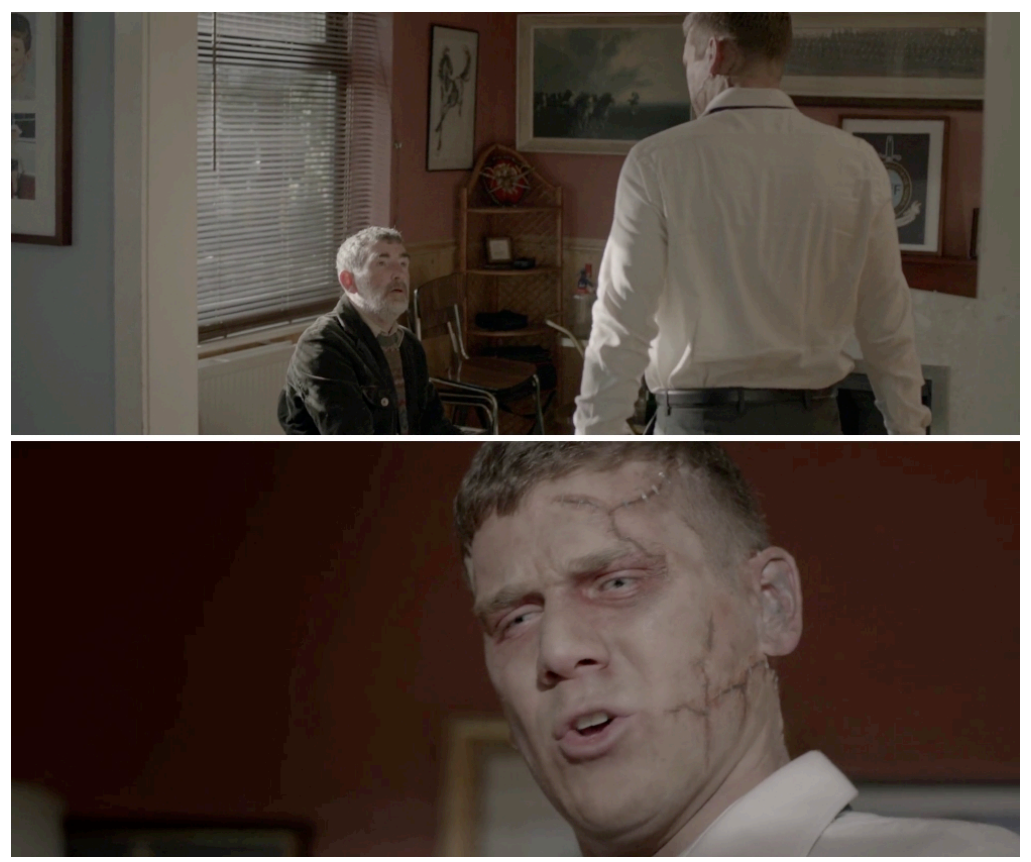

F5. In the Flesh (BBC Three: 2013-2014).

Será precisamente en este pub dónde Rick es presentado por primera vez al espectador, personaje que, al igual que Kieren, regresa a Roarton como un PDS rehabilitado por el gobierno. La principal diferencia es que su padre, cegado por la arrogancia de ser el líder de los HVF, se niega en todo momento a reconocer a Rick como un zombi, de la misma manera que se negaba en el pasado a aceptar la posible homosexualidad de su hijo, llegando a expulsar a Kieren de su casa para evitar que ambos jóvenes mantuvieran contacto. Para 
ello, Bill tratará de reforzar aquello que considera normal, es decir, realizando actividades que perpetúan los estereotipos de la masculinidad y la heterosexualidad: habla con él sobre sexo y mujeres, le lleva a un campo de tiro, y le permite beber junto a las tropas de los HVF en el pub. La actitud de Bill con su hijo parece decirnos que se puede ser homosexual siempre y cuando lo ocultes bajo un halo de heterosexualidad (normalidad), es decir, cuando no aparentes serlo. Una actitud que queda probada cuando Rick se enfrenta a su padre en el último episodio de la primera temporada, mostrándose ante él sin maquillaje y negándose a cumplir sus órdenes de asesinar a Kieren ( $\mathrm{F}_{5}$ ), porque como bien dice: "Papá. No quiero lastimar a [Kie]Ren. Es mi mejor amigo. Si [Kie]Ren es malvado, papá, entonces yo también lo soy.” El desenlace, como no podía ser de otra manera, será funesto para Rick. Su padre es incapaz de hacer frente a la verdad y termina acabando con la vida de su hijo (F6).

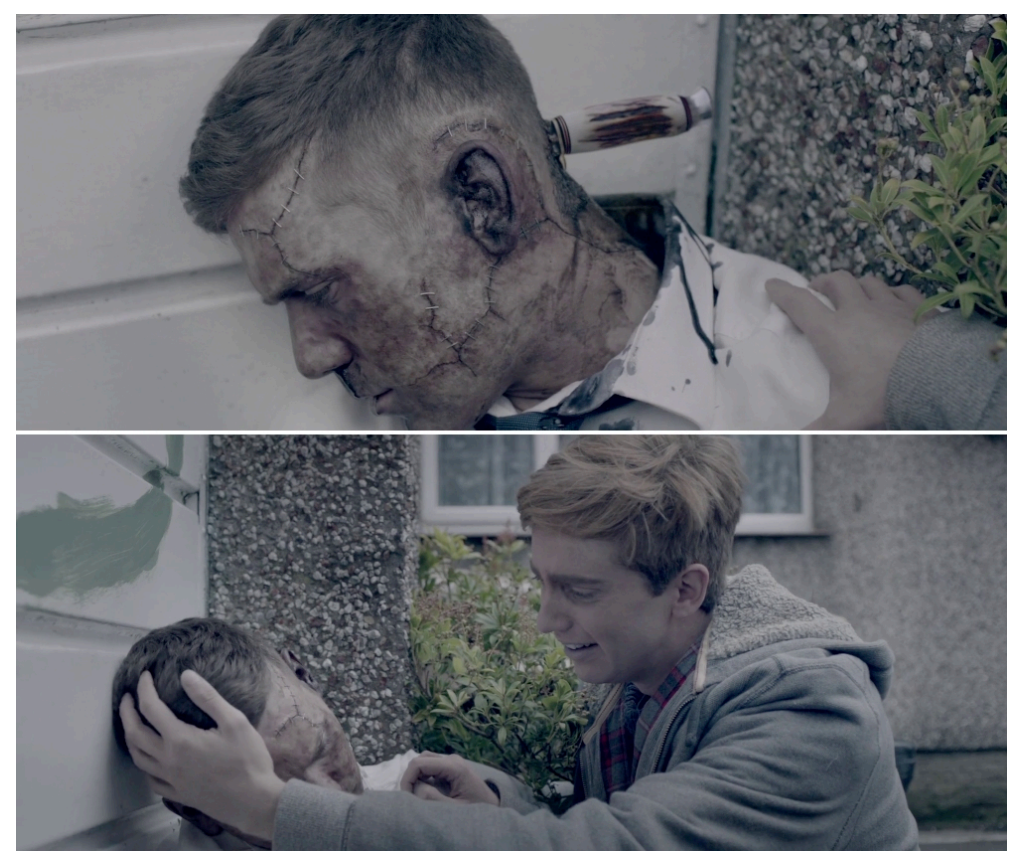

F6. In the Flesh (BBC Three: 2013-2014).

Con este fatal desenlace terminaría la primera temporada, la cual centra su foco en la historia personal de Kieren y su reintegración a la sociedad de Roarton, así como al núcleo familiar. Como hemos podido ver, la serie, partiendo de la utilización del zombi como construcción metafórica que sustituye a la figura del homosexual, explora temas como la identidad sexual 
dentro de una sociedad represiva que oprime al que no cumple los cánones de lo considerado normal. Para la segunda temporada, este conflicto va a verse potenciado por la aparición del partido político Victus, liderado en Roarton por Maxine Martin, que se opone a la reintegración social de los zombis y los reprime tomando medidas contra ellos. De tal forma que el malestar social de una parte de la población de Roarton, visto en los tres episodios que configuran la primera temporada, va a materializarse en forma de brazo político de extrema derecha. Su mensaje electoral es lo bastante claro:

Damas y caballeros, no se dejen engañar por las mentiras pregonadas por la élite corrupta. Aquellos llamados afectados por el Síndrome del parcialmente muerto no son como ustedes o como yo...con deseos y sueños normales. Puede que pretendan ser como nosotros, pero lo que hay debajo de esa máscara de maquillaje y medicación es un asesino frío y calculador con el que no se puede razonar. El PDS que está en sus casas, en sus supermercados, en sus bares, está a una dosis de distancia de querer destriparle.

Un mensaje que recuerda a las declaraciones xenófobas del presidente del partido euroescéptico de derechas UKIP Nigel Farage en contra de los rumanos asentados en Reino Unido, como señalaba Stuart Jeffries en la reseña que escribe de In the Flesh para el diario británico The Guardian (Jeffries, 2014). Resulta difícil, sin duda, no encontrar un cierto paralelismo entre el Victus de la serie y los discursos de odio propagados por los actuales partidos políticos de extrema derecha que tan en auge están en la Europa actual. Basta con destacar que este partido nace como reacción a la postura moderada y tolerante a los PDS por parte del gobierno de Inglaterra, siendo su principal medida la supresión de cualquier derecho que estos tuvieran, así como la creación de un programa de servicios comunitarios forzosos como pago por el daño causado a la sociedad durante El Levantamiento (F7).

La situación se polarizará hasta tal punto que el acoso y derribo al que son sometidos los PDS terminará dando lugar a la aparición de grupos extremistas como el Ejército de Liberación de los No-Muertos (del inglés ULA: Undead Liberation Army), el cual optará por atentar contra los humanos como forma de protesta por las políticas xenófobas del partido de Maxine Martin. En conclusión, en su segunda temporada Dominic Mitchell va a dejar en un 
segundo plano la crítica hacia la homofobia para desarrollar la narrativa y ampliar aún más las posibilidades de la metáfora zombi, con el objetivo de abordar los miedos que despiertan en la sociedad temas como el terrorismo, la radicalización o la intolerancia social por razones de género, raza o clase.

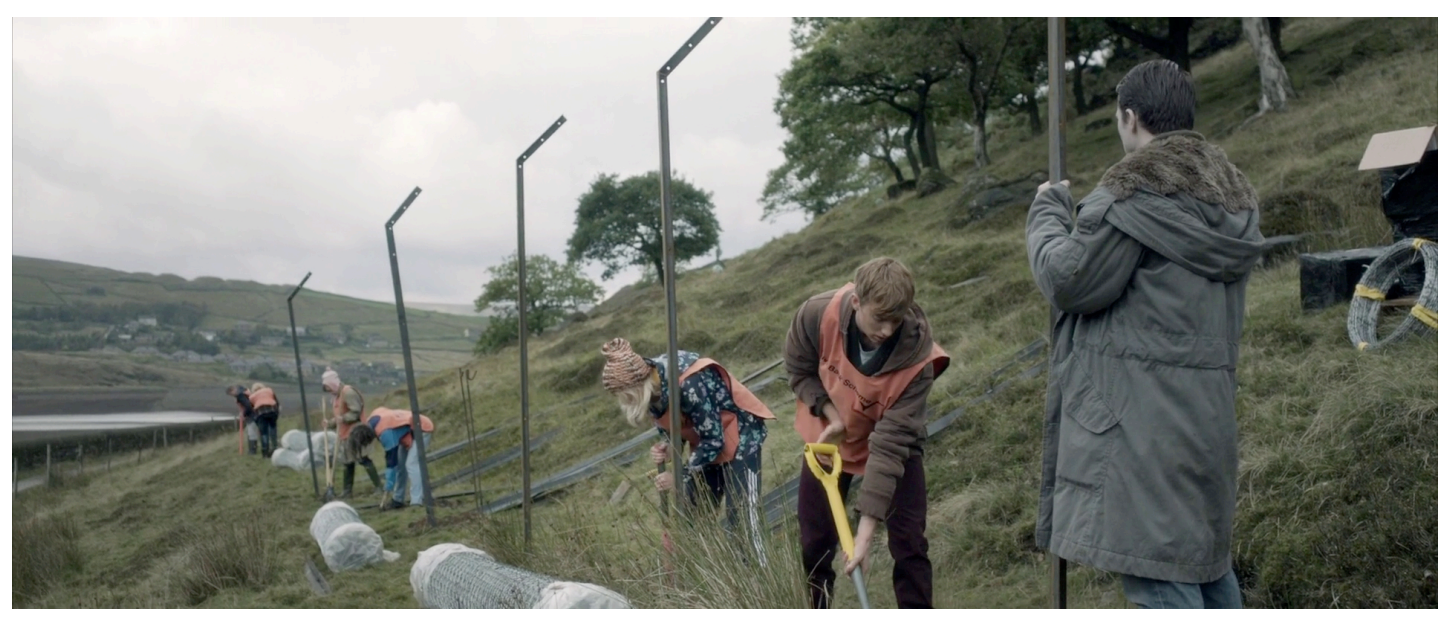

F7. In the Flesh (BBC Three: 2013-2014).

\section{Conclusiones}

La irrupción definitiva del zombi en la ficción televisiva ha sido determinante en lo que se refiere a la renovación de su uso alegórico. Decimos definitiva porque, aunque el zombi ha estado presente en el medio desde hace décadas recordemos su papel en series de animación como Los Simpson, Scooby-Doo o Shout Park- no es hasta la llegada en el año 2010 de The Walking Dead cuando esta irrupción se produce de forma contundente. El éxito abrumador de la serie creada por Robert Kirkman ha abierto el camino a historias que difícilmente pudieran ser contadas en una película. En este sentido, la televisión se ha convertido en un espacio idóneo para las narrativas zombis gracias al formato serializado, en tanto que ofrece a estas la posibilidad de desplegar la metáfora zombi a medida que la narración va desarrollándose poco a poco, de temporada a temporada.

El género, lejos de estar agotado, está más vivo que nunca gracias al boom cultural de las series de televisión. Los sistemas de distribución han cambiado en los últimos años, de tal manera que el público cada día cuenta con una mayor facilidad para ver series a las que de otra manera no hubiera 
podido acceder. En resumen, el zombi está hoy en día más presente en nuestras pantallas gracias a la multiplicidad de plataformas que ofrecen video bajo demanda y esto, a su vez, ha favorecido y facilitado el surgimiento de los primeros estudios dedicados a la metáfora zombi en España, donde hasta el momento esta cuestión no se había examinado con la misma profundidad que en el ámbito anglo-americano.

Las propuestas más interesantes, curiosamente, las encontramos en aquellas series que trabajan en torno a la humanización del muerto viviente, un rasgo que comenzaron a trabajar las comedias zombis de la década de los ochenta y que se ha demostrado fundamental en el desarrollo del género dentro de la ficción televisiva. A lo largo de este artículo mencionábamos algunas de las que mayor relevancia han alcanzado, como son Dead Set: Muerte en directo, iZombie, Les Revenants o ZombieLars. Por su parte, aunque cancelada prematuramente en su segunda temporada pese a gozar de un gran apoyo por parte de sus fans, In the Flesh es un buen ejemplo de las posibilidades que el género puede ofrecer en la televisión.

Partiendo del arquetipo creado por Romero en 1968, Dominic Mitchell consigue revitalizar la metáfora zombi haciendo uso de la humanización del muerto viviente para desarrollar aún más su potencial a la hora de elaborar críticas de carácter sociopolítico. En un conocimiento profundo de la tradición cinematográfica de esta criatura, In the Flesh avanza por caminos hasta ahora no explorados y que abren el género a nuevas lecturas que entroncan con la realidad social del momento. Así mismo, al convertir al zombi en víctima y utilizar esta condición como símbolo de la homosexualidad por la que el protagonista de la serie es despreciado, cuestiona la monstruosidad entendida como Otredad y su relación con lo normativo. Si bien en esta ocasión hemos focalizado nuestro análisis en el uso del muerto viviente como representación metafórica del colectivo LGTB y la homofobia en una sociedad fuertemente influenciada por el fundamentalismo religioso y político, la serie puede ser abordada desde otros ángulos, puesto que en ella se habla de otras cuestiones como son la pérdida de un ser querido y la superación del luto, del suicidio entre los adolescentes, etc. (Abbot, 2016b, pp. 155-171). En este sentido, creemos que la segunda temporada de la serie merece ser abordada de forma 
independiente en futuros escritos y puesta en relación con series como ZombieLars, en la que el zombi también es utilizado para elaborar una crítica en torno a la xenofobia y una reflexión sobre cómo enfrenta Noruega sus miedos y contradicciones en relación al auge de los partidos de extrema derecha.

La serie de Dominic Mitchell constituye, en definitiva, un ejemplo del brillante futuro que esta criatura tiene por delante en la televisión. Mientras que el modelo instaurado por Romero puede seguir siendo atractivo y gozar de gran éxito, In the Flesh deja claro que el futuro del zombi radica en su hibridación con otros géneros y narrativas, así como en la necesidad de contar historias desde el punto de vista del muerto viviente.

\section{Referencias bibliográficas}

Abbot, S. (2016a). Undead Apocalyse: Vampires and Zombies in the 21st Century. Edimburgo, Escocia: Edinburh University Press Ltd.

Abbot, S. (2016b). Loss is Part of the Deal: Love, Fear and Mourning in TV Horror. En A. N. García Martínez (Ed.), Emotions in Contemporary TV Series (pp.155-171). Basingstoke, Reino Unido: Palgrave Macmillan UK.

Aiossa, E. (2018). The Subversive Zombie: Social Protest and Gender in Undead Cinema and Television. North Carolina, Estados Unidos: Mcfarland \& Co Inc.

Benshoff, H. M. (1997). Monsters in the Closet: Homosexuality and the Horror Film. Manchester, Reino Unido: Manchester University Press.

Bishop, K. W. (2010). American Zombie Gothic: The Rise and Fall (and Rise) of the Walking Dead in Popular Culture. North Carolina, Estados Unidos: Mcfarland \& Co Inc.

Bishop, K. W. (2015a). I always wanted to see how the other half lives: The Contemporary Zombie as Seductive Proselyte. En L. Hubner, M. Leaning y P. Manning (Eds.), The Zombie Renaissance in Popular Culture (pp.26-38). Basingstoke, Reino Unido: Palgrave Macmillan UK.

Bishop, K. W. (2015b). How Zombies Conquered Popular Culture: The Multifarious Walking Dead in the 21st Century. North Carolina, Estados Unidos: Mcfarland \& Co Inc.

Cohen, J. J. (1996). Monster Culture (Seven Theses). En J. J. Cohen (Ed.), Monster Theory: Reading Culture (pp. 3-25). Minneapolis, Estados Unidos: University of Minnesota Press. 
Dendle, P. (2007). The Zombie as Barometer of Cultural Anxiety. En S. Niall (Ed.), Monsters and the Monstrous: Myths and Metaphors of Enduring Evil (pp.45-57). Amsterdam, Países Bajos: Rodopi.

Enríquez Veloso, G. (2012). Intertextualidad en The Simpsons: Transgresión y ruptura formal. Ponencia llevada a cabo en el 1oth World Congress of the International Association for Semiotic Studies (IASS/AIS), Coruña, España.

Jackson, C. (2017). This place. It's never going to accept people like us. Never ever: (Queer) Horror, Hatred and Heteronormativity in In the Flesh. En A. Szanter y J. K. Richards (Eds.), Romancing the Zombie: Essays on the Undead as Significant "Other" (pp.118-135). North Carolina, Estados Unidos: Mcfarland \& Co Inc.

Jeffries, S. (5 de mayo de 2014). In the Flesh; The Man in the Hat - TV review. The Guardian. Recuperado de: https://www.theguardian.com/tv-andradio/2014/may/05/in-the-flesh-tv-review

Jones, S. (2010). Implied...or Implode? The Simpsons' Carnivalesque Treehouse of Horror. Animation Journal, 18, 56-79.

Manning, P. (2015). Zombies, Zomedies, Digital Fan Cultures and the Politics of Taste. En L. Hubner, M. Leaning y P. Manning (Eds.), The Zombie Renaissance in Popular Culture (pp.160-173). Basingstoke, Reino Unido: Palgrave Macmillan UK.

Mercer, K. (1993). Monster metaphors: notes on Michael Jackson's Thriller. En S. Frith, A. Goodwin y L. Grossberg (Eds.), Sound and Vision: The Music Video Reader (pp.93-128). Londres, Reino Unido: Routledge.

O'Connell, M. (13 de octubre de 2014). The Walking Dead Sets Another Ratings Record. The Hollywood Reporter. Recuperado de: https://www.hollywoodreporter.com/live-feed/walking-dead-setsratings-record-740324

O'Connell, M. (25 de octubre de 2016). TV Ratings: 'Walking Dead' Bloodbath Matches All-Time Audience High. The Hollywood Reporter. Recuperado de: https://www.hollywoodreporter.com/live-feed/tvratings-walking-dead-bloodbath-matches-all-time-audience-high940924

Rogers, M. C., Epstein, M., y Reeves, J. L. (2002). The Sopranos as HBO Brand Equity: The Art of Commerce in the Age of Digital Reproduction. En D. Lavery (Ed.), This Thing of Ours: Investigating The Sopranos (pp.4257). New York, Estados Unidos: Columbia University Press.

Tartaglione, N. (27 de enero de 2017). Resident Evil: The Final Chapter To Push Franchise Past \$1B Global This Weekend. Deadline Hollywood. Recuperado de: https://deadline.com/2017/01/resident-evil-the-finalchapter-franchise-crossing-one-billion-dollars-global-box-office1201895482/

Tenga, A., y Zimmerman, E. (2013). Vampire Gentlemen and Zombie Beasts: A Rendering of True Monstrosity. Gothic Studies, 15(1), 76-87. https://doi.org/10.7227/GS.15.1.8 
Wood, R. (2003). Hollywood from Vietnam to Reagan and Beyond. New York, Estados Unidos: Columbia University Press. 